Vietnam Journal of Mechanics, VAST, Vol.40, No. 2 (2018), pp. $171-180$

DOI:10.15625/0866-7136/10807

\title{
REFLECTION AND TRANSMISSION OF QUASI-P WAVES THROUGH AN ORTHOTROPIC LAYER SANDWICHED BETWEEN TWO HALF-SPACES
}

\author{
Do Xuan Tung ${ }^{1, *}$, Nguyen Thi Kieu ${ }^{1}$, Luong The Thang ${ }^{2}$ \\ ${ }^{1}$ Hanoi Architectural University, Hanoi, Vietnam \\ ${ }^{2}$ Vietnam National University of Forestry, Hanoi, Vietnam \\ *E-mail: tungdx2783@gmail.com \\ Received October 16, 2017
}

\begin{abstract}
In this paper, the reflection and transmission of a $\mathrm{qP}$ wave through an orthotropic elastic layer sandwiched between two orthotropic elastic half-spaces is investigated. The main aim is to derive the expressions in closed-form of the reflection and transmission coefficients. These expressions have been obtained by using the transfer matrix for an orthotropic layer. Based on the obtained expressions, some numerical examples are carried out to examine the dependence of the reflection and transmission coefficients on the incident angle, the wave frequency and the ratio of mass densities.
\end{abstract}

Keywords: Reflection, transmission, qP wave, qSV wave, orthotropic, reflection and transmission coefficients.

\section{INTRODUCTION}

The reflection and transmission of elastic waves have long been an important research subject in various fields such as geophysics, acoustics, materials science, oil and gas exploration, design of ultrasonic transducer and so on due to its important role in practical applications. Extensive review of works on this research direction has been reported in well-known books on the elastic wave topic by Ewing et al. [1], Brekhovskikh [2], Brekhovskikh and Gordin [3], Ben-Menahem \& Singh [4], Nayfeh [5], Borcherdt [6]. Most of investigations on this topic devoted to the wave reflection and transmission in isotropic elastic media.

The wave reflection and transmission in anisotropic elastic media have attracted the attention of researchers only recently due to two reasons. Firstly, the anisotropic materials are now widely used in various fields of the modern technology. Secondly, the problems of wave reflection and transmission in anisotropic media is significantly different from that in isotropic media, such as the wave velocity depends on the wave propagation direction, for the longitudinal wave the direction of the displacement vector does not coincide with the wave propagation direction and so on. It should be noted that, the

(C) 2018 Vietnam Academy of Science and Technology 
main aim of the investigations of reflection and transmission of elastic waves is to derive expressions in closed-form of reflection and transmission coefficients.

The reflection of elastic waves at a surface of an anisotropic elastic half-space was studied by Chattopadhyay and Choudhury [7], Chatterjee et al. [8]. The reflection and transmission of elastic waves at an interface between two anisotropic elastic half-spaces was investigated by Rokhlin et al. [9], Chattopadhyay et al. [10-12]. The reflection and transmission of elastic waves through a layer sandwiched between two anisotropic elastic half-spaces, two inhomogeneous viscoelastic half-spaces [13], a more complicated problem, was investigated by Paswan and Sahu [14] for a fluid layer and by Kumari et al. [15] for an isotropic elastic layer. However, no investigation has been carried out so far for the case when the sandwiched elastic layer is anisotropic, to the best knowledge of the authors. Therefore, the main purpose of this paper is to consider the reflection and transmission of $\mathrm{qP}$ waves through an orthotropic elastic layer sandwiched between two orthotropic elastic half-spaces. In order to obtain the closed-form formulas for the reflection and transmission coefficients, the transfer matrix for an orthotropic elastic layer, established recently by Vinh et al. [16], Tuan and Trung [17], is employed. Based on the obtained formulas, some numerical examples are carried out to examine the dependence of the reflection and transmission coefficients on the incident angle, the incident wave frequency and the ratio of mass densities.

\section{REFLECTION AND TRANSMISSION COEFFICIENTS}

Consider an orthotropic elastic layer of thickness $h$ sandwiched between two distinct orthotropic elastic half-spaces $\Omega^{+}$and $\Omega^{-}$as shown in Fig. 1 . Here we use the orthogonal coordinate system $0 x_{1} x_{2} x_{3}$ in which the $x_{2}$-axis is perpendicular to the layer. The layer, the upper half-space $\Omega^{+}$and the lower half-space $\Omega^{-}$occupy the domain $0 \leq x_{2} \leq h$, $x_{2} \leq 0$ and $x_{2} \geq h$, respectively (see Fig. 1 ). The material constants, mass density of the layer and two half-spaces are given by

$$
c_{i j}, \rho= \begin{cases}c_{i j}^{+}, \rho^{+} & \text {for } \quad x_{2} \leq 0 \\ c_{i j}^{-}, \rho^{-} & \text {for } \quad x_{2} \geq h \\ \overline{c_{i j}}, \bar{\rho} & \text { for } \quad 0 \leq x_{2} \leq h\end{cases}
$$

It is assumed that the principal material planes of the orthotropic layer and of two orthotropic half-spaces are identical and coincide with the coordinate planes. We are interested in the plain strain so that

$$
u_{i}=u_{i}\left(x_{1}, x_{2}, t\right), i=1,2, \quad u_{3} \equiv 0 .
$$

Suppose that an incident quasi $P$ wave ( $q P$ wave) with the amplitude $\mathbf{R}_{0}\left(R_{01}, R_{02}\right)$ propagates in the upper half-space $\Omega^{+}$and it is of the form $[7,11,18]$

$$
\left(\begin{array}{l}
u_{01} \\
u_{02}
\end{array}\right)=\left(\begin{array}{l}
R_{01} \\
R_{02}
\end{array}\right) \exp \left[i k_{0}\left(x_{1} p_{01}+x_{2} p_{02}-c_{0} t\right)\right]
$$

where $\theta_{0}$ is the incident angle, $p_{01}=\sin \theta_{0}, p_{02}=\cos \theta_{0}$ are components of the unit propagation vector, $c_{0}$ is the velocity of wave and $k_{0}=\omega / c_{0}$ is the wave number, $\omega$ 


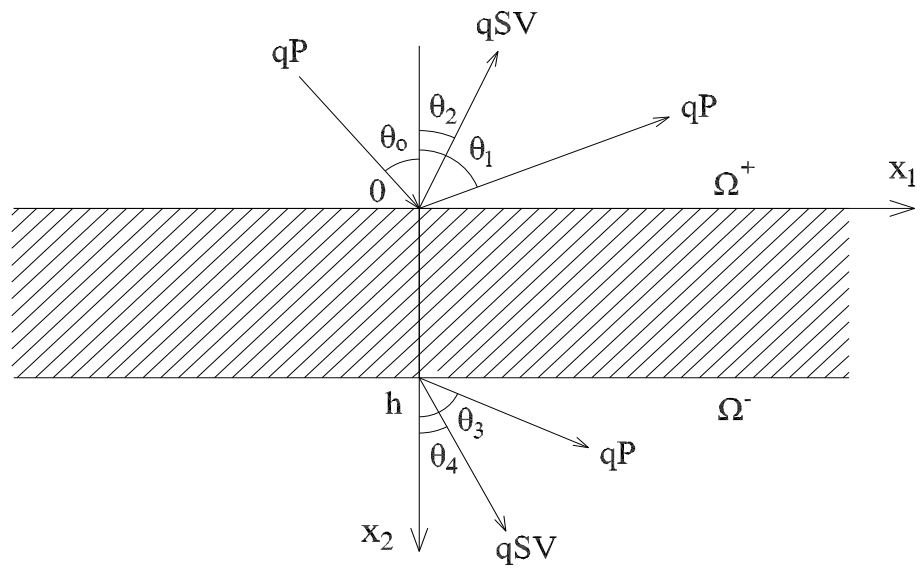

Fig. 1. An orthotropic elastic layer sandwiched between two orthotropic elastic half-spaces

is the circular frequency that is given. The velocity $c_{0}$ of the incident $q P$ wave is given by $[7,11,18]$

$$
2 \rho c_{0}^{2}=\left(M_{0}+P_{0}\right)+\left[\left(M_{0}-P_{0}\right)^{2}+4\left(N_{0}\right)^{2}\right]^{1 / 2},
$$

in which

$$
M_{0}=c_{11}^{+} p_{01}^{2}+c_{66}^{+} p_{02}^{2}, \quad N_{0}=\left(c_{66}^{+}+c_{12}^{+}\right) p_{01} p_{02}, \quad P_{0}=c_{66}^{+} p_{01}^{2}+c_{22}^{+} p_{02}^{2} .
$$

When the $q P$ wave strikes the layer it generates reflected $\mathrm{qP}, \mathrm{qSV}$ waves propagating in the half-space $\Omega^{+}$and transmitted $q P, q S V$ waves traveling in the half-space $\Omega^{-}$(see Fig. 1). The displacement fields of these waves are of the form $[7,11,18]$ as follows:

For the reflected $q P$ wave with amplitude $\mathbf{R}_{1}\left(R_{11}, R_{12}\right)$

$$
\left(\begin{array}{l}
u_{11} \\
u_{12}
\end{array}\right)=\left(\begin{array}{l}
R_{11} \\
R_{12}
\end{array}\right) \exp \left[i k_{1}\left(x_{1} p_{11}+x_{2} p_{12}-c_{1} t\right)\right] .
$$

For the reflected $q S V$ wave with amplitude $\mathbf{R}_{2}\left(R_{21}, R_{22}\right)$

$$
\left(\begin{array}{l}
u_{21} \\
u_{22}
\end{array}\right)=\left(\begin{array}{l}
R_{21} \\
R_{22}
\end{array}\right) \exp \left[i k_{2}\left(x_{1} p_{21}+x_{2} p_{22}-c_{2} t\right)\right] .
$$

For the transmitted $q P$ wave with amplitude $\mathbf{R}_{3}\left(R_{31}, R_{32}\right)$ :

$$
\left(\begin{array}{l}
u_{31} \\
u_{32}
\end{array}\right)=\left(\begin{array}{l}
R_{31} \\
R_{32}
\end{array}\right) \exp \left[i k_{3}\left(x_{1} p_{31}+x_{2} p_{32}-c_{3} t\right)\right] .
$$

For the transmitted $q S V$ wave with amplitude $\mathbf{R}_{4}\left(R_{41}, R_{42}\right)$

$$
\left(\begin{array}{l}
u_{41} \\
u_{42}
\end{array}\right)=\left(\begin{array}{l}
R_{41} \\
R_{42}
\end{array}\right) \exp \left[i k_{4}\left(x_{1} p_{41}+x_{2} p_{42}-c_{4} t\right)\right],
$$

where $p_{n 1}, p_{n 2},(n=1,2,3,4)$ are components of the unit propagation vectors of the reflected waves $q P, q S V$, the transmitted waves $q P, q S V$, respectively, see Fig.1, that are determined by Singh et al [18], $k_{i}, c_{i}$ are the wave numbers, the wave velocities whose 
relation is: $\omega=k_{0} c_{0}=k_{1} c_{1}=k_{2} c_{2}=k_{3} c_{3}=k_{4} c_{4}$. The velocities $c_{n}(n=1,2,3,4)$ are determined as follows $[7,11,18]$.

a) For the longitudinal waves ( $q P$ waves)

$$
2 \rho c_{n}^{2}=\left(M_{n}+P_{n}\right)+\left[\left(M_{n}-P_{n}\right)^{2}+4\left(N_{n}\right)^{2}\right]^{1 / 2}, n=1,3 .
$$

b) For the transverse waves ( $q S V$ waves)

$$
2 \rho c_{n}^{2}=\left(M_{n}+P_{n}\right)-\left[\left(M_{n}-P_{n}\right)^{2}+4\left(N_{n}\right)^{2}\right]^{1 / 2}, n=2,4 .
$$

The expressions $M_{n}, N_{n}, P_{n}$ for upper half-space $(n=1,2)$ are

$$
M_{n}=c_{11}^{+} p_{n 1}^{2}+c_{66}^{+} p_{n 2}^{2}, \quad N_{n}=\left(c_{66}^{+}+c_{12}^{+}\right) p_{n 1} p_{n 2}, \quad P_{n}=c_{66}^{+} p_{n 1}^{2}+c_{22}^{+} p_{n 2}^{2} .
$$

The expressions $M_{n}, N_{n}, P_{n}$ for lower half-space $(n=3,4)$ are similar to (12) in which the signs " $(+)$ " are replaced by signs " $(-)$ ".

In Eqs (6)-(9), the components $R_{i 1}, R_{i 2}$ of amplitudes $\mathbf{R}_{i}$ are needed to be determined. In addition, they have relations $[7,11,18]$

$$
F_{i}=\frac{R_{i 1}}{R_{i 2}}=\frac{N_{i}}{\rho^{+} c_{i}^{2}-M_{i}}=\frac{\rho^{+} c_{i}^{2}-P_{i}}{N_{i}} ; \quad\left|\mathbf{R}_{i}\right|=\sqrt{R_{i 1}^{2}+R_{i 2}^{2}} \quad i=0,1,2,3,4 .
$$

The reflection, transmission coefficients of reflected $q P, q S V$ waves and transmitted $q P, q S V$ waves are defined as

$$
R_{q P}=\frac{\left|\mathbf{R}_{1}\right|}{\left|\mathbf{R}_{0}\right|}=\frac{\sqrt{1+F_{1}^{2}}}{\sqrt{1+F_{0}^{2}}} \frac{\left|R_{12}\right|}{\left|R_{02}\right|} ; \quad R_{q S V}=\frac{\left|\mathbf{R}_{2}\right|}{\left|\mathbf{R}_{0}\right|}=\frac{\sqrt{1+F_{2}^{2}}}{\sqrt{1+F_{0}^{2}}} \frac{\left|R_{22}\right|}{\left|R_{02}\right|},
$$

for the reflected waves and

$$
T_{q P}=\frac{\left|\mathbf{R}_{3}\right|}{\left|\mathbf{R}_{0}\right|}=\frac{\sqrt{1+F_{3}^{2}}}{\sqrt{1+F_{0}^{2}}} \frac{\left|R_{32}\right|}{\left|R_{02}\right|} ; \quad T_{q S V}=\frac{\left|\mathbf{R}_{4}\right|}{\left|\mathbf{R}_{0}\right|}=\frac{\sqrt{1+F_{4}^{2}}}{\sqrt{1+F_{0}^{2}}} \frac{\left|R_{42}\right|}{\left|R_{02}\right|},
$$

for the transmitted waves.

\section{FORMULAS IN CLOSED-FORM FOR THE REFLECTION AND TRANSMISSION COEFFICIENTS}

In order to determine the reflection and transmission coefficients the transfer matrix for an orthotropic elastic layer derived recently by Vinh et al. [16] is used.

From (3)-(7), the displacement field of the upper half-space $\Omega^{+}$is expressed as

$$
\begin{aligned}
& u_{1}^{+}=u_{01}+u_{11}+u_{21}=e^{i\left(\eta x_{1}-\omega t\right)}\left(R_{01} e^{\left(i k_{0} p_{02} x_{2}\right)}+R_{11} e^{\left(i k_{1} p_{12} x_{2}\right)}+R_{21} e^{\left(i k_{2} p_{22} x_{2}\right)}\right), \\
& u_{2}^{+}=u_{02}+u_{12}+u_{22}=e^{i\left(\eta x_{1}-\omega t\right)}\left(R_{02} e^{\left(i k_{0} p_{02} x_{2}\right)}+R_{12} e^{\left(i k_{1} p_{12} x_{2}\right)}+R_{22} e^{\left(i k_{2} p_{22} x_{2}\right)}\right),
\end{aligned}
$$

where $\eta$ is the horizontal wave-number.

Similarly, from (8) and (9), the displacement field of lower half-space $\Omega^{-}$is given by

$$
\begin{aligned}
& u_{1}^{-}=u_{31}+u_{41}=e^{i\left(\eta x_{1}-\omega t\right)}\left(R_{31} e^{\left(i k_{3} p_{32} x_{2}\right)}+R_{41} e^{\left(i k_{4} p_{42} x_{2}\right)}\right), \\
& u_{2}^{-}=u_{32}+u_{42}=e^{i\left(\eta x_{1}-\omega t\right)}\left(R_{32} e^{\left(i k_{3} p_{32} x_{3}\right)}+R_{42} e^{\left(i k_{4} p_{42} x_{3}\right)}\right) .
\end{aligned}
$$


From Snell's law we have the following identities [11,19]

$$
\eta=k_{0} p_{01}=k_{1} p_{11}=k_{2} p_{21}=k_{3} p_{31}=k_{4} p_{41} \text {. }
$$

The constitutive equations for orthotropic material are of form [20]

$$
\sigma_{11}=c_{11} u_{1,1}+c_{12} u_{2,2}, \quad \sigma_{22}=c_{12} u_{1,1}+c_{22} u_{2,2}, \quad \sigma_{12}=c_{66}\left(u_{2,1}+u_{1,2}\right) .
$$

From (16), (17) and (19), the stress components $\sigma_{11}^{+}, \sigma_{12}^{+}, \sigma_{22}^{+}$and $\sigma_{11}^{-}, \sigma_{12}^{-}, \sigma_{22}^{-}$of the upper and the lower half-spaces are derived.

We introduce a new unknown vector given by $\boldsymbol{\xi}\left(x_{2}\right)=\left[u_{1}, u_{2}, \sigma_{12}, \sigma_{22}\right]^{T}$. By $\xi^{+}, \xi^{-}$ and $\xi^{L}$ we denote the corresponding values of $\xi$ for the upper half-space, the lower halfspace and the layer respectively. Suppose that the layer and the half-spaces are perfectly bonded to each other. Then, the displacements and stresses are required to be continuous through the interfaces $x_{2}=0$ and $x_{2}=h$, i. e.

$$
\xi^{+}(0)=\xi^{L}(0), \quad \xi^{-}(h)=\xi^{L}(h)
$$

where

$$
\xi^{+}(0)=\left[\begin{array}{c}
u_{1}^{+}(0) \\
u_{2}^{+}(0) \\
\sigma_{12}^{+}(0) \\
\sigma_{22}^{+}(0)
\end{array}\right], \quad \boldsymbol{\xi}^{-}(h)=\left[\begin{array}{c}
u_{1}^{-}(h) \\
u_{2}^{-}(h) \\
\sigma_{12}^{-}(h) \\
\sigma_{22}^{-}(h)
\end{array}\right],
$$

and (we assign $R_{i 2}=B_{i}, i=0,1,2,3,4$ )

$$
\begin{aligned}
u_{1}^{+}(0)= & F_{0} B_{0}+F_{1} B_{1}+F_{2} B_{2} ; \quad u_{2}^{+}(0)=B_{0}+B_{1}+B_{2}, \\
\sigma_{12}^{+}(0)= & c_{66}^{+} i\left(F_{0} B_{0} k_{0} p_{02}+F_{1} B_{1} k_{1} p_{12}+F_{2} B_{2} k_{2} p_{22}+B_{0} k_{0} p_{01}+B_{1} k_{1} p_{11}+B_{2} k_{2} p_{21}\right), \\
\sigma_{22}^{+}(0)= & i\left(c_{12}^{+}\left(F_{0} B_{0} k_{0} p_{01}+F_{1} B_{1} k_{1} p_{11}+F_{2} B_{2} k_{2} p_{21}\right)\right. \\
& \left.+c_{22}^{+}\left(B_{0} k_{0} p_{02}+B_{1} k_{1} p_{12}+B_{2} k_{2} p_{22}\right)\right), \\
u_{1}^{-}(h)= & F_{3} B_{3} \cdot \exp \left(i k_{3} p_{32} h\right)+F_{4} B_{4} \cdot \exp \left(i k_{4} p_{42} h\right), \\
u_{2}^{-}(h)= & B_{3} \cdot \exp \left(i k_{3} p_{32} h\right)+B_{4} \cdot \exp \left(i k_{4} p_{42} h\right), \\
\sigma_{12}^{-}(h)= & c_{66}^{-} i\left(F_{3} B_{3} k_{3} p_{32} \cdot \exp \left(i k_{3} p_{32} h\right)+F_{4} B_{4} k_{4} p_{42} \cdot \exp \left(i k_{4} p_{42} h\right)\right. \\
& \left.+B_{3} k_{3} p_{31} \cdot \exp \left(i k_{3} p_{32} h\right)+B_{4} k_{4} p_{41} \cdot \exp \left(i k_{4} p_{42} h\right)\right), \\
\sigma_{22}^{-}(h)= & c_{12}^{-} i\left(F_{3} B_{3} k_{3} p_{31} \cdot \exp \left(i k_{3} p_{32} h\right)+F_{4} B_{4} k_{4} p_{41} \cdot \exp \left(i k_{4} p_{42} h\right)\right) \\
& +c_{22}^{-} i\left(B_{3} k_{3} p_{32} \cdot \exp \left(i k_{3} p_{32} h\right)+B_{4} k_{4} p_{42} \cdot \exp \left(i k_{4} p_{42} h\right)\right) .
\end{aligned}
$$

On the other hand, using the transfer matrix of an orthotropic layer we have [16]

$$
\xi^{L}(0)=\mathbf{T} \xi^{L}(h)
$$


in which

$$
\mathbf{T}=\left[\begin{array}{cccc}
\frac{[\bar{\gamma} ; \mathrm{ch} \varepsilon]}{[\bar{\gamma}]} & \frac{-i[\bar{\beta} ; \mathrm{sh} \varepsilon]}{[\bar{\alpha} ; \bar{\beta}]} & \frac{-[\bar{\alpha} ; \mathrm{sh} \varepsilon]}{[\bar{\alpha} ; \bar{\beta}]} & \frac{-i[\mathrm{ch} \varepsilon]}{[\bar{\gamma}]} \\
\frac{-i[\bar{\gamma} ; \bar{\alpha} \mathrm{sh} \varepsilon]}{[\bar{\gamma}]} & \frac{[\bar{\alpha} \mathrm{ch} \varepsilon ; \bar{\beta}]}{[\bar{\alpha} ; \bar{\beta}]} & \frac{-i \bar{\alpha}_{1} \bar{\alpha}_{2}[\mathrm{ch} \varepsilon]}{[\bar{\alpha} ; \bar{\beta}]} & \frac{-[\bar{\alpha} \mathrm{sh} \varepsilon]}{[\bar{\gamma}]} \\
\frac{-[\gamma ; \bar{\beta} \mathrm{sh} \varepsilon]}{[\bar{\gamma}]} & \frac{-i \bar{\beta}_{1} \bar{\beta}_{2}[\mathrm{ch} \varepsilon]}{[\bar{\alpha} ; \bar{\beta}]} & \frac{[\bar{\alpha} ; \bar{\beta} \mathrm{ch} \varepsilon]}{[\bar{\alpha} ; \bar{\beta}]} & \frac{i[\bar{\beta} \mathrm{sh} \varepsilon]}{[\bar{\gamma}]} \\
\frac{-i \bar{\gamma}_{1} \bar{\gamma} 2[\mathrm{ch} \varepsilon]}{[\bar{\gamma}]} & \frac{[\bar{\beta} ; \bar{\gamma} \mathrm{sh} \varepsilon]}{[\bar{\alpha} ; \bar{\beta}]} & \frac{-i[\bar{\alpha} ; \bar{\gamma} \mathrm{sh} \varepsilon]}{[\bar{\alpha} ; \bar{\beta}]} & \frac{[\bar{\gamma} \mathrm{ch} \varepsilon]}{[\bar{\gamma}]}
\end{array}\right],
$$

is the transfer matrix of orthotropic layer given by Vinh et al. [16] and

$$
[f ; g]:=f_{2} g_{1}-f_{1} g_{2},[f]:=f_{2}-f_{1},
$$

denote the jump of quantities inside bracket. Furthermore,

$$
\begin{aligned}
& c=c_{0} / \sin \left(\theta_{0}\right), \quad k=k_{0} \sin \left(\theta_{0}\right), \quad \varepsilon=k h, \\
& \bar{\alpha}_{k}=-\frac{\left(\bar{c}_{12}+\bar{c}_{66}\right) \bar{b}_{k}}{\bar{c}_{22} \bar{b}_{k}^{2}-\bar{c}_{66}+\bar{X}}, k=1,2, \bar{X}=\bar{\rho} c^{2} \\
& \bar{b}_{1}=\sqrt{\frac{\bar{S}+\sqrt{\bar{S}^{2}-4 \bar{P}}}{2}}, \bar{b}_{2}=\sqrt{\frac{\bar{S}-\sqrt{\bar{S}^{2}-4 \bar{P}}}{2}}, \\
& \bar{S}=\frac{\bar{c}_{22}\left(\bar{c}_{11}-\bar{X}\right)+\bar{c}_{66}\left(\bar{c}_{66}-\bar{X}\right)-\left(\bar{c}_{12}+\bar{c}_{66}\right)^{2}}{\bar{c}_{22} \bar{c}_{66}}, \\
& \bar{P}=\frac{\left(\bar{c}_{11}-\bar{X}\right)\left(\bar{c}_{66}-\bar{X}\right)}{\bar{c}_{22} \bar{c}_{66}}, \\
& \bar{\beta}_{n}=\bar{c}_{66}\left(\bar{b}_{n}-\bar{\alpha}_{n}\right), \bar{\gamma}_{n}=\bar{c}_{12}+\bar{c}_{22} \bar{b}_{n} \bar{\alpha}_{n}, n=1,2 .
\end{aligned}
$$

The components $t_{i j}$ of the transfer matrix $T$ have properties [16]

$$
t_{11}=t_{33}, t_{12}=t_{43}, t_{14}=t_{23}, t_{21}=t_{34}, t_{22}=t_{44}, t_{32}=t_{41} .
$$

Using (20), (23), and taking into account (21), (24), we have

$$
\left[\begin{array}{l}
u_{1}^{+}(0) \\
u_{2}^{+}(0) \\
\sigma_{12}^{+}(0) \\
\sigma_{22}^{+}(0)
\end{array}\right]=\left[\begin{array}{llll}
t_{11} & t_{12} & t_{13} & t_{14} \\
t_{21} & t_{22} & t_{23} & t_{24} \\
t_{31} & t_{32} & t_{33} & t_{34} \\
t_{41} & t_{42} & t_{43} & t_{44}
\end{array}\right]\left[\begin{array}{l}
u_{1}^{-}(h) \\
u_{2}^{-}(h) \\
\sigma_{12}^{-}(h) \\
\sigma_{22}^{-}(h)
\end{array}\right] .
$$

Therefore, we have 4 equations for 4 unknowns $B_{1} ; B_{2} ; B_{3} ; B_{4}$ (taking into account (22)), namely

$$
\left\{\begin{array}{l}
a_{1} B_{1}+a_{2} B_{2}+a_{3} B_{3}+a_{4} B_{4}=a_{5}, \\
a_{6} B_{1}+a_{7} B_{2}+a_{8} B_{3}+a_{9} B_{4}=a_{10}, \\
a_{11} B_{1}+a_{12} B_{2}+a_{13} B_{3}+a_{14} B_{4}=a_{15}, \\
a_{16} B_{1}+a_{17} B_{2}+a_{18} B_{3}+a_{19} B_{4}=a_{20} .
\end{array}\right.
$$


This equation system leads to

$$
\left(\begin{array}{l}
B_{1} \\
B_{2} \\
B_{3} \\
B_{4}
\end{array}\right)=\left[\begin{array}{llll}
a_{1} & a_{2} & a_{3} & a_{4} \\
a_{6} & a_{7} & a_{8} & a_{9} \\
a_{11} & a_{12} & a_{13} & a_{14} \\
a_{16} & a_{17} & a_{18} & a_{19}
\end{array}\right]^{-1}\left(\begin{array}{c}
a_{5} \\
a_{10} \\
a_{15} \\
a_{20}
\end{array}\right)
$$

where

$$
\begin{aligned}
& a_{1}=-F_{1} ; a_{2}=-F_{2} ; a_{5}=F_{0} B_{0} ; a_{6}=a_{7}=-1 ; a_{10}=B_{0} ; a_{15}=c_{66}^{+} i B_{0}\left(F_{0} p_{02}+p_{01}\right), \\
& a_{3}=\left(t_{11} F_{3}+t_{12}+t_{13} c_{66}^{-} i k_{3}\left(F_{3} p_{32}+p_{31}\right)+t_{14} i k_{3}\left(c_{12}^{-} F_{3} p_{31}+c_{22}^{-} p_{32}\right)\right) \exp \left(i k_{3} p_{32} h\right), \\
& a_{4}=\left(t_{11} F_{4}+t_{12}+t_{13} c_{66}^{-} i k_{4}\left(F_{4} p_{42}+p_{41}\right)+t_{14} i k_{4}\left(c_{12}^{-} F_{4} p_{41}+c_{22}^{-} p_{42}\right)\right) \exp \left(i k_{4} p_{42} h\right), \\
& a_{8}=\left(t_{21} F_{3}+t_{22}+t_{14} c_{66}^{-} i k_{3}\left(F_{3} p_{32}+p_{31}\right)+t_{24} i k_{3}\left(c_{12}^{-} F_{3} p_{31}+c_{22}^{-} p_{32}\right)\right) \exp \left(i k_{3} p_{32} h\right), \\
& a_{9}=\left(t_{21} F_{4}+t_{22}+t_{14} c_{66}^{-} i k_{4}\left(F_{4} p_{42}+p_{41}\right)+t_{24} i k_{4}\left(c_{12}^{-} F_{4} p_{41}+c_{22}^{-} p_{42}\right)\right) \exp \left(i k_{4} p_{42} h\right), \\
& a_{11}=c_{66}^{+} i k_{1}\left(F_{1} B_{1} p_{12}+B_{1} p_{11}\right) ; a_{12}=c_{66}^{+} i k_{2}\left(F_{2} B_{2} p_{22}+B_{2} p_{21}\right), \\
& a_{13}=\left(t_{31} F_{3}+t_{11}+t_{14} c_{66}^{-} i k_{3}\left(F_{3} p_{32}+p_{31}\right)+t_{21} i k_{3}\left(c_{12}^{-} F_{3} p_{31}+c_{22}^{-} p_{32}\right)\right) \exp \left(i k_{3} p_{32} h\right), \\
& a_{14}=\left(t_{31} F_{4}+t_{32}+t_{11} c_{66}^{-} i k_{4}\left(F_{4} p_{42}+p_{41}\right)+t_{21} i k_{4}\left(c_{12}^{-} F_{4} p_{41}+c_{22}^{-} p_{42}\right)\right) \exp \left(i k_{4} p_{42} h\right), \\
& a_{16}=c_{12}^{+} i k_{1}\left(F_{1} B_{1} p_{11}+B_{1} p_{12}\right) ; a_{17}=c_{12}^{+} i k_{2}\left(F_{2} B_{2} p_{21}+B_{2} p_{22}\right), \\
& a_{18}=\left(t_{32} F_{3}+t_{42}+t_{12} c_{66}^{-} i k_{3}\left(F_{3} p_{32}+p_{31}\right)+t_{22} i k_{3}\left(c_{12}^{-} F_{3} p_{31}+c_{22}^{-} p_{32}\right)\right) \exp \left(i k_{3} p_{32} h\right), \\
& a_{19}=\left(t_{32} F_{4}+t_{42}+t_{12} c_{66}^{-} i k_{4}\left(F_{4} p_{42}+p_{41}\right)+t_{22} i k_{4}\left(c_{12}^{-} F_{4} p_{41}+c_{22}^{-} p_{42}\right)\right) \exp \left(i k_{4} p_{42} h\right), \\
& a_{20}=i k_{0} B_{0}\left(c_{12}^{+} F_{0} p_{01}+c_{22}^{+} p_{02}\right) .
\end{aligned}
$$

For an incident $q P$ wave, $\theta_{0}$ is given and, therefore, $c_{0}\left(\theta_{0}\right)$ is computed by (4). The terms $p_{i 1}=\cos \theta_{i} ; p_{i 2}=\sin \theta_{i} ; k_{i}$ and $F_{i}$ are computed by (18). The detail of procedure for the calculation of other quantities is represented in papers [7, 11, 16]. Finally, the reflection, transmission coefficients are calculated by (14), (15).

The formulas (14), (15), in which $R_{i 2} \equiv B_{i}$ given by (30), are closed-form expressions for the reflection, transmission coefficients.

\section{NUMERICAL RESULTS AND DISCUSSION}

In this section, the influence of incident angle, wave frequency, ratio of mass density on the reflection and transmission coefficients is illustrated. From (14), (15) we can see that the reflection and transmission coefficients depend on dimensionless parameters defined as

$$
\begin{aligned}
& e_{11}=\frac{c_{11}^{+}}{c_{66}^{+}} ; e_{21}=\frac{c_{22}^{+}}{c_{66}^{+}} ; e_{31}=\frac{c_{12}^{+}}{c_{66}^{+}} ; e_{12}=\frac{c_{11}^{-}}{c_{66}^{-}} ; e_{22}=\frac{c_{22}^{-}}{c_{66}^{-}} ; e_{32}=\frac{c_{12}^{-}}{c_{66}^{-}}, \\
& e_{1}=\frac{\bar{c}_{11}}{\bar{c}_{66}} ; e_{2}=\frac{\bar{c}_{22}}{\bar{c}_{66}} ; e_{3}=\frac{\bar{c}_{12}}{\bar{c}_{66}} ; r=\frac{\rho^{-}}{\rho^{+}} ; r_{\mu}=\frac{c_{66}^{-}}{c_{66}^{+}} ; r_{L}=\frac{\bar{\rho}}{\rho^{+}} ; r_{\mu L}=\frac{\bar{c}_{66}}{c_{66}^{+}} ; \epsilon=k_{0} h .
\end{aligned}
$$


As an example, we choose a model with the dimensionless parameters (being not relative to any particular material) as

$$
\begin{aligned}
& e_{11}=33.8333 ; e_{21}=40.8333 ; e_{31}=12.5000 ; e_{12}=2.4787 ; e_{22}=2.9255, \\
& e_{32}=0.8511 ; e_{1}=2.9999 ; e_{2}=2.9999 ; e_{3}=0.9999 ; r=1.5745 ; r_{\mu}=15.6667, \\
& r_{L}=0.0017 ; r_{\mu L}=48.3750 ; \epsilon=1 .
\end{aligned}
$$

In Fig. 2, the modulus of amplitudes of reflected, transmitted waves against the incident angle $\theta_{0}$ for $\epsilon=1$ are plotted. It can be seen from Fig. 2 that:

i) The modulus $R_{q P}$ is almost unchange for $\theta_{0}<50^{0}$ then increases strongly when the incident angle tends to $90^{\circ}$. Conversely, the modulus $T_{q P}$ decreases sharply at $\theta_{0}=45^{0}$ from value 0.85 to value 0 .

ii) In domain of $20^{\circ}<\theta_{0}<70^{0}$, the reflected qSV wave is dominant over $q P$ wave.

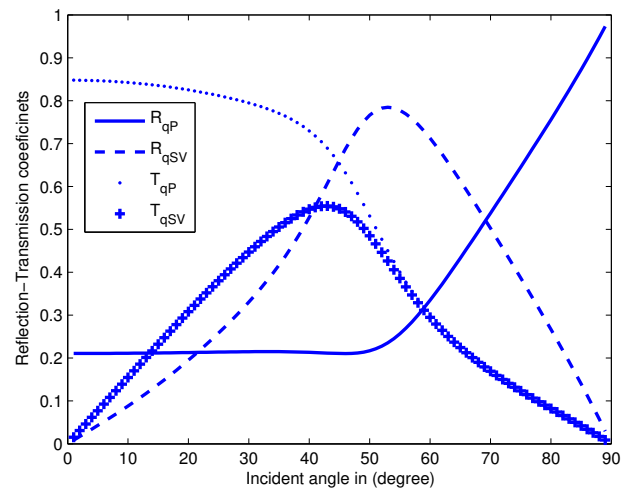

Fig. 2. The reflection, transmission coefficients as a function of incident angles

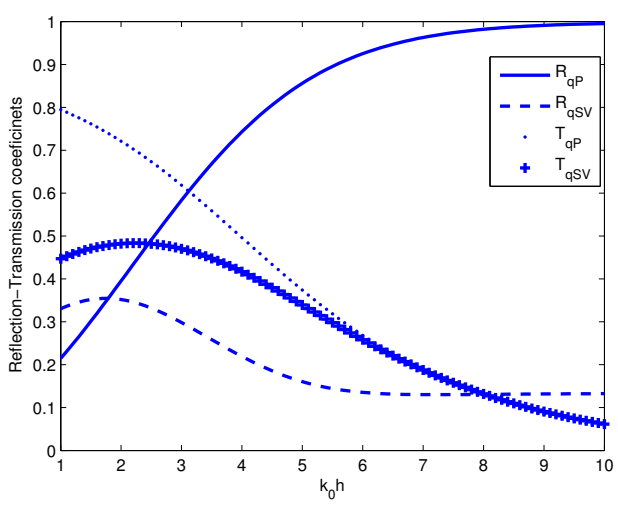

Fig. 3. The reflection, transmission coefficients as a function of wave frequency

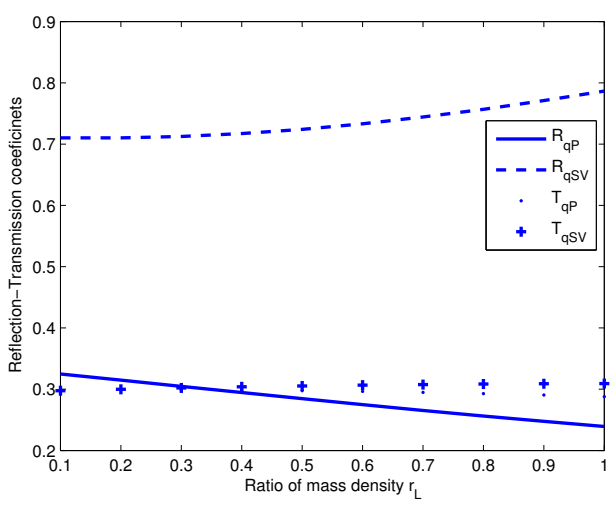

Fig. 4. The reflection, transmission coefficients as a function of ratio of mass density

Fig. 3 depicts the variation of modulus of amplitudes of reflected and transmitted waves with respect to the wave frequency $\epsilon$ when $\theta_{0}=\pi / 6$. We observed that the 
amplitude $R_{q P}$ increases in range $1 \leq \epsilon \leq 10$ while $T_{q P}$ decreases. The modulus of reflection, transmission coefficients of $q S V$ waves decrease in $1 \leq \epsilon \leq 10$.

The dependence of amplitudes of reflected, transmitted waves on $r_{L}$ with $\epsilon=1$ and $\theta_{0}=\pi / 3$ is represented in Fig. 4 . It is shown that in domain $0<r_{L} \leq 1$ the reflected $q P$ wave is dominant.

\section{CONCLUSIONS}

In this paper, problem of the reflection and transmission of a $\mathrm{qP}$ wave through an orthotropic layer sandwiched between two orthotropic elastic half-spaces is investigated and the closed-form expressions for the reflection, transmission coefficients have been obtained by using the transfer matrix for an orthotropic elastic layer. As an illustration of the application of obtained expressions, numerical results are carried to indicate the dependence of the reflection, transmission coefficients on the incident angle, wave frequency and ratio of mass density. It is shown that the reflection and transmission coefficients depend strongly on the incident angle, wave frequency and considerably on the ratio of mass density.

\section{ACKNOWLEDGMENTS}

The work was supported by the Vietnam National Foundation for Science and Technology Development (NAFOSTED) under Grant No. 107.02-2014.17.

\section{REFERENCES}

[1] W. M. Ewing and W. S. Jardetzky. Waves in layered media. McGraw-Hill, New York, (1957).

[2] L. Brekhovskikh. Waves in layered media. Academic Press, New York, second edition, (1976).

[3] L. M. Brekhovskikh and O. A. Godin. Acoustics of layered media, Vol. 10. Springer, Berlin, (2013).

[4] A. Ben-Menahem and S. J. Singh. Seismic waves and sources. Springer-Verlag, New York, (1981).

[5] A. H. Nayfeh. Wave propagation in layered anisotropic media: With application to composites, Vol. 39. NorthHolland Pub. Co., (1995).

[6] R. D. Borcherdt. Viscoelastic waves in layered media. Cambridge University Press, New York, (2009).

[7] A. Chattopadhyay and S. Choudhury. The reflection phenomena of P-waves in a medium of monoclinic type. International Journal of Engineering Science, 33, (2), (1995), pp. 195-207. doi:10.1016/0020-7225(94)00055-o.

[8] M. Chatterjee, S. Dhua, S. A. Sahu, and A. Chattopadhyay. Reflection in a highly anisotropic medium for three-dimensional plane waves under initial stresses. International Journal of Engineering Science, 85, (2014), pp. 136-149. doi:10.1016/j.ijengsci.2014.08.010.

[9] S. I. Rokhlin, T. K. Bolland, and L. Adler. Reflection and refraction of elastic waves on a plane interface between two generally anisotropic media. The Journal of the Acoustical Society of America, 79, (4), (1986), pp. 906-918. doi:10.1121/1.393764.

[10] A. Chattopadhyay, S. Saha, and M. Chakraborty. Reflection and transmission of shear waves in monoclinic media. International Journal for Numerical and Analytical Methods in Geomechanics, 21, (7), (1997), pp. 495-504. doi:10.1002/(sici)1096-9853(199707)21:7<495::aidnag884>3.0.co;2-a. 
[11] A. Chattopadhyay and Rajneesh. Reflection and refraction of waves at the interface of an isotropic medium over a highly anisotropic medium. Acta Geophysica, 54, (3), (2006), pp. 239 249. doi:10.2478/s11600-006-0022-y.

[12] A. Chattopadhyay, P. Kumari, and V. K. Sharma. Reflection and refraction at the interface between distinct generally anisotropic half spaces for three-dimensional plane quasi-P waves. Journal of Vibration and Control, 21, (3), (2015), pp. 493-508. doi:10.1177/1077546313488615.

[13] S. Kumar, P. C. Pal, and S. Majhi. Reflection and transmission of plane SH-waves through an anisotropic magnetoelastic layer sandwiched between two semi-infinite inhomogeneous viscoelastic half-spaces. Pure and Applied Geophysics, 172, (10), (2015), pp. 2621-2634. doi:10.1007/s00024-015-1048-3.

[14] B. Paswan, S. A. Sahu, and A. Chattopadhyay. Reflection and transmission of plane wave through fluid layer of finite width sandwiched between two monoclinic elastic half-spaces. Acta Mechanica, 227, (12), (2016), pp. 3687-3701. doi:10.1007/s00707-016-1684-4.

[15] P. Kumari, V. K. Sharma, and C. Modi. Reflection/refraction pattern of quasi-(P/SV) waves in dissimilar monoclinic media separated with finite isotropic layer. Journal of Vibration and Control, 22, (11), (2016), pp. 2745-2758. doi:10.1177/1077546314548911.

[16] P. C. Vinh, V. T. N. Anh, and N. T. K. Linh. On a technique for deriving the explicit secular equation of Rayleigh waves in an orthotropic half-space coated by an orthotropic layer. Waves in Random and Complex Media, 26, (2), (2016), pp. 176-188. doi:10.1080/17455030.2015.1132859.

[17] T. T. Tuan and T. N. Trung. The dispersion of Rayleigh waves in orthotropic layered half-space using matrix method. Vietnam Journal of Mechanics, 38, (1), (2016), pp. 27-38. doi:10.15625/0866-7136/38/1/6191.

[18] S. J. Singh and S. Khurana. Reflection and transmission of P and SV waves at the interface between two monoclinic elastic half-spaces. Proceedings National Academy of Sciences India Section A, 71, (4), (2001), pp. 305-320.

[19] J. Achenbach. Wave propagation in elastic solids, Vol. 16. North-Holland Publishing Company, Amsterdam-New York-Oxford, (1973).

[20] T. C. T. Ting. Anisotropic elasticity: Theory and applications, number 45. Oxford University Press, New York, (1996). 